\title{
Primary Hydatid Cyst of Spleen: A Rare Entity
}

\section{Veena Gupta, Vaanika Kaira, Jyoti Sharma, Rajeev Sen and Ashok Sangwaiya*}

Department of Pathology, Post Graduate Institute of Medical Sciences, Rohtak, Haryana, India

\begin{abstract}
Hydatid disease, a zoonosis, occurs worldwide but its prevalence is high in those countries where sheep and cattle raising constitute an important industry due to close association between man, sheep and dog. Most common sites of involvement by hydatid disease are liver followed by lung. Splenic involvement by hydatid disease is very rare accounting for only $0.9 \%$ to $8.0 \%$ of all cases. Other rare sites include heart, pancreas and muscles. We report a case of histopathologically confirmed primary hydatid cyst spleen in an 18 year old male, which is a rare entity. Hydatid disease should be considered in the differential diagnosis of cystic lesions of spleen.
\end{abstract}

Keywords: Hydatid disease; Spleen

\section{Introduction}

Splenic involvement in hydatid disease is uncommon, representing less than $2 \%$ of all human infestations by Echinococcus [1]. It is an endemic disease in the sheep and cattle raising countriesMiddle East, North Africa, New Zealand, Australia, and South America [2]. In India, the recorded prevalence of the splenic hydatid cyst is $2.5 \%$, with the highest incidence reported in the central parts [3]. In areas of endemic hydatid disease, most of these cysts are due to the larval form of this parasite, whereas non-parasitic cysts comprise the vast majority in Western countries [4].The first case of a splenic hydatid cyst was reported by Berlot in 1790 from an autopsy [5]. Herein, we report a case of splenic hydatid disease in a 18 year old male form Haryana (India). We report this case because of its rarity.

\section{Case Report}

An 18 year old male presented to surgery outpatient department with complaint of pain in left hypochondrium for one month. Patient was asymptomatic otherwise.Clinical examination revealed mild splenomegaly. Patient's haematological and serological parameters were within normal limits. Contrast Enhanced CT scan (CECT) revealed a large cystic lesion in distal portion of spleen with imperceptible wall of size approximately $9.8 \times 10 \times 8.4$ $\mathrm{cm}$. All other abdominal and pelvic organs were unremarkable on ultrasonography. Partial splenectomy with cyst removal was performed. Histopathological examination showed brood capsules with hooklets along with the cyst wall having an outer acellular laminated layer and inner germinal layer characteristic of a hydatid

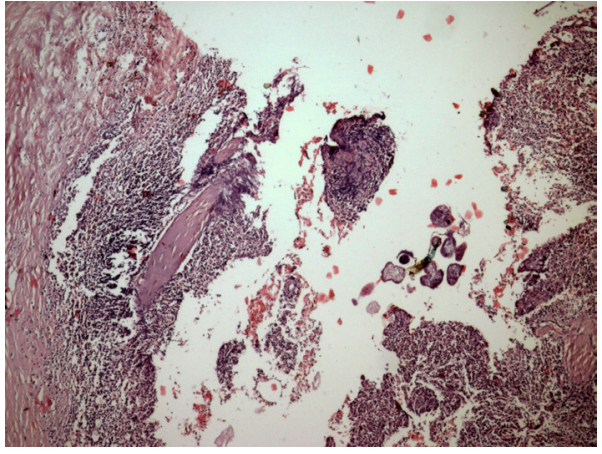

Figure 1: H \& E section showing hydatid cyst along with portion of spleen (100X). cyst (Figure1-3). A final diagnosis of splenic hydatid cyst was made. After one week patient was discharged with instructions to continue

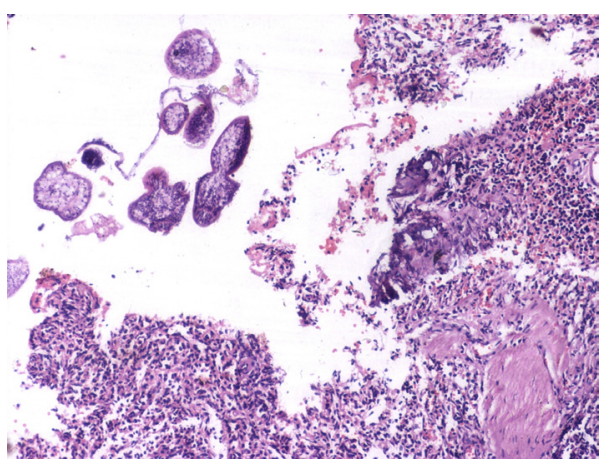

Figure 2: $\mathrm{H}$ \& $\mathrm{E}$ section showing hydatid cyst along with portion of spleen (200X)

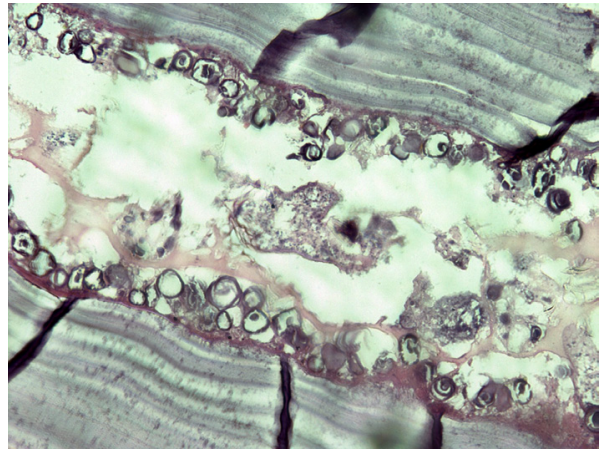

Figure 3: H \& E section showing laminated cyst wall along with scolices (400X).

*Corresponding author: Ashok Sangwaiya, Department of Pathology, Post Graduate Institute of Medical Sciences, Rohtak, Haryana, India, Tel: 9416162494 E-mail: ashoksangwaiya@gmail.com

Received February 03, 2014; Accepted February 20, 2014; Published February 22, 2014

Citation: Gupta V, Kaira V, Sharma J, Sen R, Sangwaiya A (2014) Primary Hydatid Cyst of Spleen: A Rare Entity. J Trop Dis 2: 131. doi: 10.4172/2329-891X.1000131

Copyright: @ 2014 Gupta V, et al. This is an open-access article distributed under the terms of the Creative Commons Attribution License, which permits unrestricted use, distribution, and reproduction in any medium, provided the original author and source are credited. 
a regimen of antihelminthic treatment consisting of albendazole (400 mg twice a day).

\section{Discussion}

Hydatid disease (Echinococcosis) is a zoonotic infection caused by the larval form of parasites of tapeworm, Echinococcus granulosus.

Humans are the accidental intermediate host in the development cycle of hydatid disease [2]. Four species of Echinococcus cause infection in humans; Echinococcusgranulosus and Echinococcusmultilocularis are the most common, causing cystic Echinococcosis and alveolar Echinococcosis respectively. The two other species,

E. vogeli and E. oligarthrus cause polycystic echinococcosis and are less frequently associated with human infection [6].

Although hydatid disease affects any organ or soft tissue, it most frequently found in liver (60-70\%), lungs (30\%), and rarely encountered in the kidney, spleen, bone, thyroid, breast and pancreas [2].Parasitic cysts of the spleen are almost exclusively hydatid cysts. In endemic areas, $50-80 \%$ of splenic cysts are echinococcal [7]. Splenic echinococcal cysts may be primary or secondary to ruptured liver cysts [8]. Possible routes of primary hydatid of spleen include arterial route after passing through liver (first filter) and lung (second filter). Another route is the venous route through portal circulation bypassing liver and lung. Secondary hydatid spleen usually follows systemic dissemination or intraperitoneal spread following ruptured hepatic hydatid cyst [9].

Hydatid cysts are usually slow growing and in some organs, especially in the spleen, they can reach an enormous size and be asymptomatic [1]. Splenic cysts present with local or referred pain (often postural) or signs and symptoms relating to splenomegaly, abdominal distension and compression of nearby structures [4]. However, secondary infection, cyst rupture and anaphylactic shock may develop [1].

The differential diagnosis includes cystic lesions of adjacent organs, e.g. Pancreas, liver and omentum, intrasplenic aneurysm and benign and malignant splenic tumors [4].

At present sonography and CT, are the most valuable imaging techniques for the diagnosis and evaluation of focal splenic diseases [1] the intradermal (Casoni) test is a valuable diagnostic procedure [9]. Serological tests are highly sensitive and specific for Echinococcosis [2]. More recently, immuno electrophoresis has improved diagnostic accuracy in up to $95 \%$ of cases [8]. In our case, imaging studies were inconclusive and histopathological examination confirmed the diagnosis. On histopathology, the hydatid cyst consists of three layers. The outermost adventitia (pseudo cyst) is formed of compressed splenic tissue, a middle layer laminated membrane of friable ectocyst and an innermost germinal layer, endocyst [2].

Total splenectomy, partial splenectomy, cyst enucleation and unroofing with omentoplasty are the various preferred surgical techniques to treat splenic hydatid disease [4]. In our case, partial splenectomy was preferred as the cyst was involving the distal portion and also patient was a young male. However, during surgical treatment extreme caution must be taken to avoid life threatening complications like anaphylactic shock due to spillage of cyst contents.Laparoscopic approach has also been advocated for uncomplicated hydatid cyst of the spleen. Chemotherapy and newer methods, such as Puncture, Aspiration, Injection, and Re-aspiration (PAIR) technique using hypertonic saline or $0.5 \%$ silver nitrate solutions before opening the cavities tends to kill the daughter cysts [2].

Albendazole is an effective adjuvant therapy in the treatment of hydatid cyst. There are less chances of recurrence in patient who received albendazole therapy, as we prescribed in our case [2]. Following treatment, hydatid cysts are best followed sonographically due to the absence of ionizing radiation and ease of access to this modality [7].

\section{Conclusion}

Splenic hydatid cyst should be kept in mind in patients presenting with left upper abdominal pain, especially from endemic area. An early diagnosis is recommended to avoid further complications.

\section{References}

1. Franquet T, Montes M, Lecumberri FJ, Esparza J, Bescos JM (1990) Hydatid disease of the spleen: Imaging findings in nine patients. AJR 154: 525-528.

2. Pukar MM, Pukar SM (2013) Giant solitary hydatid cyst of spleen-A case report. Int J Surg Case Rep 4: 435-437.

3. Makkar M, Gupta C, Singh DP, Kaur S, Mahajan N (2012) Giant isolated splenic hydatidosis. Trop Parasitol 2: 74-76.

4. Williams RJ, Glazer G (1993) Splenic cysts: changes in diagnosis, treatment and aetiological concepts. Ann R Coll Surg Engl 75: 87-89.

5. Muro J, Ortiz-Vázquez J, Miño G, Sanmartín P (1969) Angiographic localization of hydatid cyst of the spleen. Rev Clin Esp 115: 433-438.

6. Moro P, Schantz PM (2009) Echinococcosis: a review. Int J Infect Dis 13: 125133.

7. Rasheed K, Zargar SA, Telwani AA (2013) Hydatid cyst of spleen: a diagnostic challenge. N Am J Med Sci 5: 10-20.

8. Al-Mohaya S, Al-Awami M, Vaidya MP, Knox-Macaulay H (1986) Hydatid cyst of the spleen. Am J Trop Med Hyg 35: 995-999.

9. Torbati M (1972) Hydatid disease of the spleen. Report of an unusual case. $\mathrm{Br}$ J Surg 59: 489-491. 